\title{
Level-1 Data Driver Card of the ATLAS New Small Wheel Upgrade Compatible with the Phase II $1 \mathrm{MHz}$ Readout Scheme
}

\author{
Panagiotis Gkountoumis \\ National Technical University of Athens, Brookhaven National Laboratory \\ Email: panagiotis.gkountoumis@cern.ch \\ On behalf of the ATLAS Muon Collaboration
}

\begin{abstract}
The Level-1 Data Driver Card (L1DDC) will be designed for the needs of the future upgrades of the innermost stations of the ATLAS end-cap muon spectrometer. The L1DDC is a high speed aggregator board capable of communicating with a large number of front-end electronics. It collects the Level-1 data along with monitoring data and transmits them to a network interface through a single bidirectional fiber link. In addition, the L1DDC board distributes trigger, time and configuration data coming from the network interface to the front-end boards. The L1DDC is fully compatible with the Phase II upgrade where the trigger rate is expected to reach $1 \mathrm{MHz}$.

This paper describes the overall scheme of the data acquisition process and especially the L1DDC board. Finally, the electronics layout on the chamber is also mentioned.
\end{abstract}

\section{INTRODUCTION}

The ATLAS New Small Wheel (NSW) upgrade is motivated by the high background radiation expected during Run-3 (2021 - 2023) and ultimately luminosity of $7 \times 10^{34} \mathrm{~cm}^{-2} \mathrm{~s}^{-1}$ in HL-LHC (after 2026). The number of interactions per bunchcrossing (each $25 \mathrm{~ns}$ ) will be increased up to 140 , resulting in a dramatically large amount of produced data. In the ATLAS experiment [1] the present muon Small Wheels will be replaced by the NSW. The NSW is a set of precision tracking and trigger detectors able to work at high rates with excellent real-time spatial and time resolution. The new detectors consist of the resistive Micromegas (MM) and the small Strip Thin Gap Chambers (sTGC) [2].

Furthermore, a radiation dose up to 1700 Gy (inner radius) and a magnetic field up to $0.4 \mathrm{~T}$ in the end cap region, create a hostile environment for the front-end electronics. To read out the high number of electronics channels and in order to survive in such a harsh environment new electronics must be fabricated and installed. In addition, correction mechanisms for Single Event Upsets (SEU - this is a change of state caused by a high-energy particle strike to a micro-electronic device) must be implemented to assure the integrity of the transmitted data.

The Level-1 Data Driver Card (L1DDC) is an intermediate board that aggregates and transmits the Level-1 data (time, charge and strip address corresponding to a single hit) from multiple front-end (FE) boards to a network interface called Front End LInk eXchange (FELIX) [3]. This is achieved using a high speed serializer/deserializer Aplication Specific Inte- grated Circuit (ASIC) called GigaBit Transceiver (GBTX) [4] developed at CERN. In general, the L1DDC combines three distinct paths: Timing, Trigger and Control (TTC) [5] data, Data Acquisition and Slow Control information, into one bidirectional optical link at a rate of $4.8 \mathrm{Gbps}$, as shown in Figure 1.

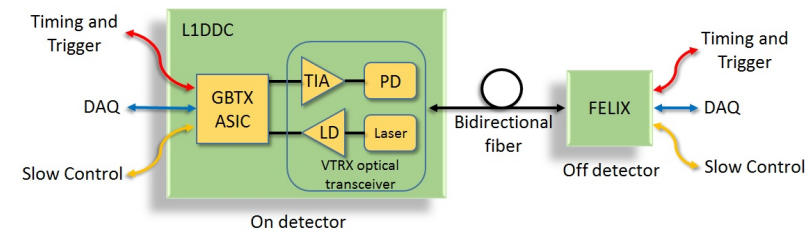

Fig. 1: The L1DDC, which resides on the detector and is implemented with custom ASICs, combines the three district paths (Timing and Trigger, DAQ, Slow Control) into a single bidirectional optical link that it will be processed by FELIX.

\section{Connectivity}

The L1DDC resides on the detector and interfaces with the FEs and for the MM case also with the Address in Real Time (ART) Data Driver Card (ADDC) [6] boards. The FE boards contain a number of ASICs called VMMs [7] each providing trigger and tracking primitives for 64 detector channels. A second ASIC, the Read Out Controller (ROC) will aggregate, process and format the data generated by the VMM FE chips. The ROC will aggregates the raw data streams from up to eight VMMs, filter the data based on the Level-1 Bunch Crossing ID (BCID) and transmit the data to the L1DDC through serial streams. Moreover, ROC receives the TTC data and the Level1 trigger from the L1DDC. For configuring and monitoring the VMMs and ROC a third ASIC called the Slow Control Adapter (SCA) will be used on the FEs. The ADDC boards collect directly from the VMMs the ART data which provide the strip information for the first arrival hit in the MM detectors.

Because of the different characteristics of both detector technologies, different $\mathrm{FE}$ boards will be fabricated for $\mathrm{MM}$ and sTGC detectors. For the MM detectors eight FE (MMFE8) [8] will be connected to one L1DDC contrary to the sTGC detectors where only three FE will be connected to one L1DDC. 
In the other direction the interface with the FELIX is made through a CERN-custom-made optical transceiver called Versatile Transceiver (VTRx) as shown in Figure 2.

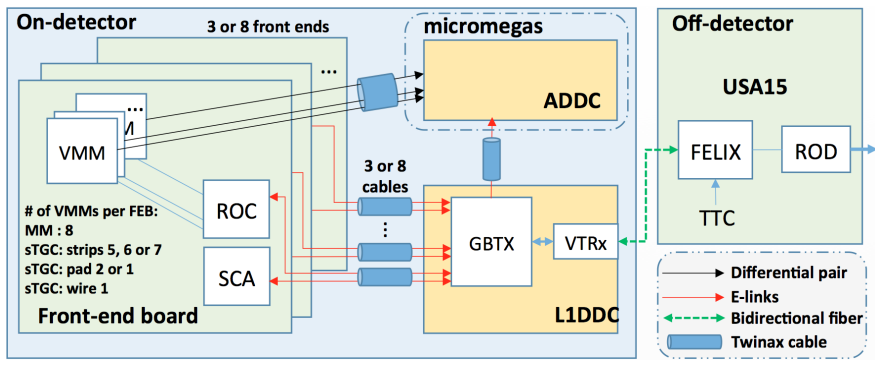

Fig. 2: Scheme of NSW electronics trigger and dataflow. The L1DDC board is connected to the ROC and the SCA ASICs of the FE boards and also to the ADDC board. Depending on the detector technology L1DDC is connected with eight FEs for the MM and with three for the sTGC detectors. The L1DDC also interfaces with the off-detector electronics and especially with the FELIX interface through a bidirectional fiber.

\section{FUNCTIONALITY}

The GBTX ASIC and subsequently the L1DDC is capable of multiplexing a number of serial links (e-links) to a single fiber. One e-Link, consists of three differential pairs (6 wires) being the clock (Clk+ and Clk-), the data in (Din+ and Din-) and the data out (Dout+ and Dout-). On the transmitting side data and clock have the same relative phase [4]. The GBTX ASIC can support up to 40 e-Links divided into five groups called banks, as shown in Figure 3. Each bank can support up to eight e-Links at $80 \mathrm{Mbps}$, four e-Links at $160 \mathrm{Mbps}$ or two e-Links at $320 \mathrm{Mbps}$. The transmitting data use the Double Data Rate (DDR) signaling.

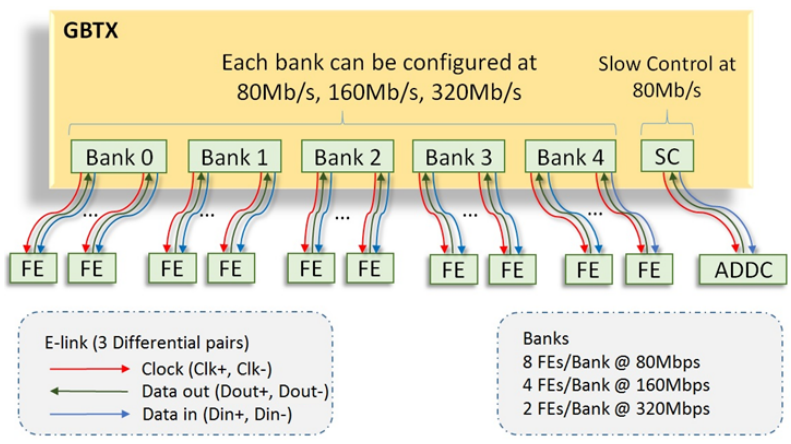

Fig. 3: GBTX e-Link connectivity. There are five banks which support up to $40 \mathrm{FE}$ boards. In each bank eight FE boards can be connected at $80 \mathrm{Mbps}$, four at $160 \mathrm{Mbps}$ or two at $320 \mathrm{Mbps}$. An extra Slow Control e-Link with a fixed rate at $80 \mathrm{Mbps}$ is used for the connection to the ADDC board.

For the interconnection of the on-detector electronics the mini Serial Attached SCSI (Small Computer System Interface) (miniSAS) cables will be used. The L1DDC is connected to the FE board through a single miniSAS cable that carries two
e-Links and a small number of differential pairs. Among these two e-Links, one is connected to the ROC ASIC and the other e-Link to the SCA ASIC, as shown in Figure 2.

Generally, the data rate on the FE boards decreases by moving radially away from the beam line. Moreover, data rate simulations showed that for $1 \mathrm{MHz}$ trigger rate the bit rate will exceed $320 \mathrm{Mbps}$ for the inner FE boards. For this reason, a second GBTx ASIC and consequently a second VTRx will be used. Having more available e-Links, a special configuration scheme will be used to achieve higher rates. At least one eLink with $320 \mathrm{Mbps}$ will be used for all FE boards and a number of differential pairs will be multiplexed to different GBTX banks. With this configuration the fifth spare bank of the one GBTX is used for the communication with the SCA ASICs of each MMFE at the $80 \mathrm{Mbps}$ data rate. A maximum of $62.9 \%$ bandwidth utilization for each readout pair is achieved as shown in Figure 4.

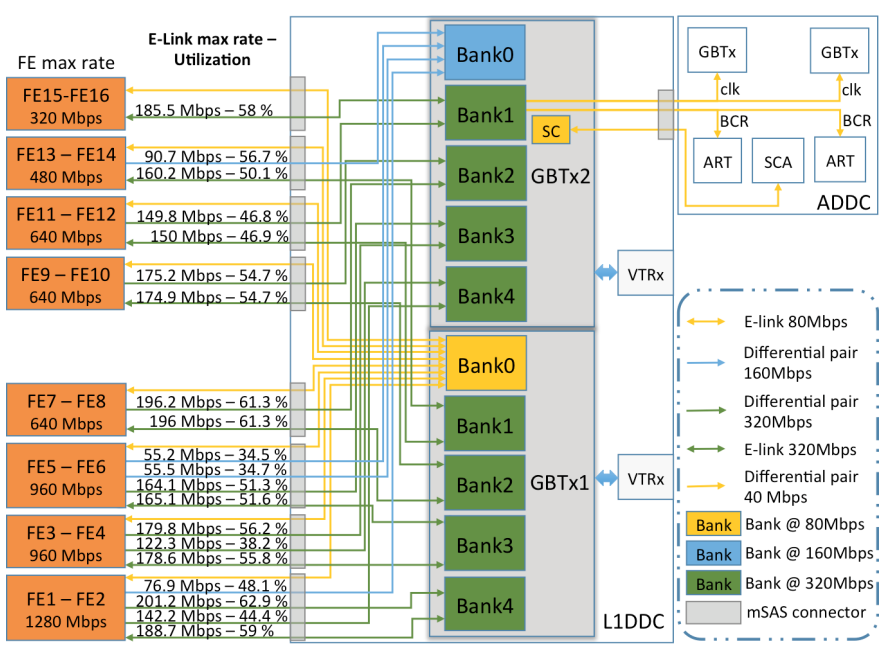

Fig. 4: MM e-Link connectivity. For the most FEs additional differenial pairs will be used to collect the Level-1 data. These pairs will be multiplexed in different banks and may use different e-Link speeds. Eight GBTX banks will run at $320 \mathrm{Mbps}$, one at $160 \mathrm{Mbps}$ and one at $80 \mathrm{Mbps}$ which will be used for the configuration of the VMM and ROC ASICs.

The GBTX ASIC has an extra Slow Control (SC) e-Link with a fixed rate at $80 \mathrm{Mbps}$ for slow control information. This extra e-Link will be used for the connection to the ADDC boards. Except the configuration data L1DDC provides the clock and Bunch Crossing Reset (BCR) signals to the ADDC board. For the transmission of these signals four unused transmitting pairs will be used from a GBTX bank.

\section{A. LIDDC board description}

Because of the different FE characteristics, different L1DDC boards will be fabricated for MM and sTGC detectors. Both boards will use the same components, with the difference that in $\mathrm{MM}$ detectors nine connectors will be used (eight for the FEs and one for the ADDC) contrary to the sTGC detectors where only three connectors will be used. The size of the 
L1DDC board for the MM detectors will be $200 \mathrm{~mm}$ in length, $60 \mathrm{~mm}$ in width and $12 \mathrm{~mm}$ in height as shown in Figure 5. The size of the L1DDC for the sTGC detectors will be $180 \mathrm{~mm}$ in length, $60 \mathrm{~mm}$ in width and $18 \mathrm{~mm}$ in height as shown in Figure 6.

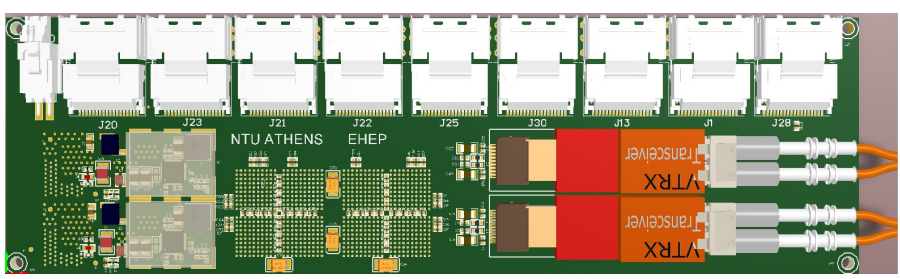

(a) Top side of the board: The nine miniSAS connectors (top), the power connector (top left), the shields of the FEAST DC-DC converters (bottom left) and the VTRX optical transceiver (bottom right) are visible.

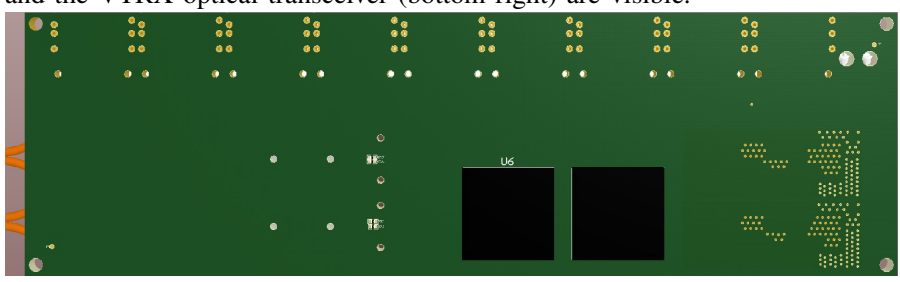

(b) Bottom side of the board: The GBTX ASIC and the position pins are visible. The VTRx and the DC-DC converters will be cooled from the bottom side of the board.

Fig. 5: A 3D representation of the L1DDC board for the MM detectors.

As mentioned before, the miniSAS cables will be used for the connection of the on-detector electronics. The high flexibility of these cables, which can support up to ten differential pairs, and the small size of the 36 position connectors makes them suitable for the boards.

\section{B. ASIC description}

The GBTX is a full radiation tolerant ASIC fabricated using the IBM/GlobalFoundries $130 \mathrm{~nm}$ CMOS technology. Its power supply is $1.5 \mathrm{~V}$ and its power consumption is $2.2 \mathrm{~W}$ in full operation. E-Links use Scalable Low-Voltage Signalling (SLVS) for $400 \mathrm{mV}$ (SLVS-400) [9]. The SLVS is a differential standard with a swing of $200 \mathrm{mV}$, centered on $0.2 \mathrm{~V}$. The GBTX ASIC has a Clock and Data Recovery (CDR) circuit which receives high speed serial data from the VTRx. It recovers and generates an appropriate high speed clock to correctly sample the incoming data stream. The serial data is then de-serialized and decoded, with appropriate error corrections, and finally DeSCRambled (DSCR). In the transmitter part the data are SCRambled (SCR), to obtain DC balance, and then encoded with a Forward Error Correction (FEC) code before being serialized and sent to the optical transceiver.

The GBTX has registers for permanent storage that are called e-fuses. Initial configuration information is taken from the e-fuses, which can then be modified via the optical link itself or via an $\mathrm{I}^{2} \mathrm{C}$ slave interface.

The VTRx optical transceiver consist of two radiation tolerant ASICs: the GigaBit TransImpendance Amplifier (GB-

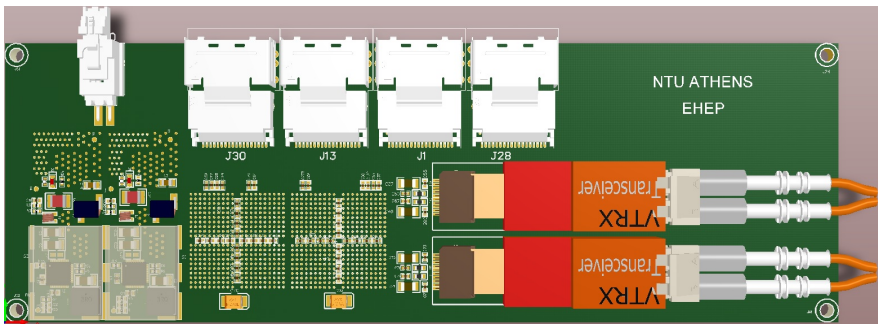

(a) Top side of the board: the three miniSAS connectors (top), the shields of the FEAST DC-DC (bottom left), the power connector (top left) and the VTRX transceiver (right) are visible.

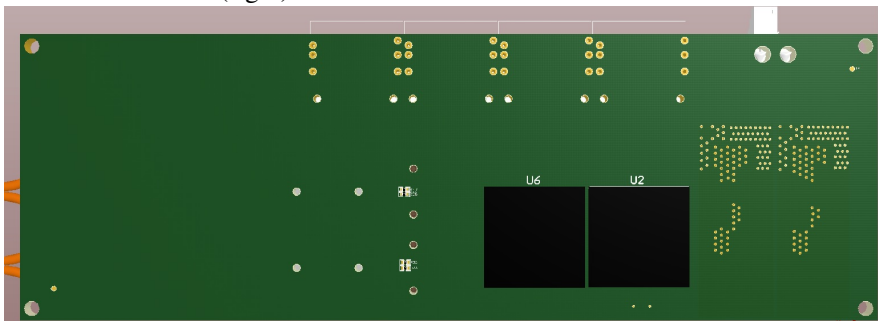

(b) Bottom side of the board: the GBTX ASIC is visible. The FEAST ASIC and the VTRx will be attached to the cooling channel from the bottom side of the board.

Fig. 6: A 3D representation of the L1DDC for the sTGC detectors.

TIA) [10], [11] and the GigaBit Laser Diode (GBLD) [12]. The GBTIA has a bit rate of 5 Gbps (min) and a total jitter smaller than $40 \mathrm{ps}$. Its supply voltage is $2.5 \mathrm{~V}$ and its power consumption is $250 \mathrm{~mW}$. The GBLD is a radiation tolerant ASIC also fabricated in $130 \mathrm{~nm}$ technology. It has also a bit rate of $5 \mathrm{Gbps}$ or more, supply voltage of $2.5 \mathrm{~V}$ and its power consumption is about $325 \mathrm{~mW}$ [12]. The VTRx is the largest component on the L1DDC board with a width of $45.3 \mathrm{~mm}$, a length of $14.5 \mathrm{~mm}$ and a height of $10 \mathrm{~mm}$.

According to the detector power distribution scheme the FEAST DC-DC converter [13] is used. This is also a custom DC-DC converter fabricated at CERN and has an input voltage range from $5 \mathrm{~V}$ to $12 \mathrm{~V}, 4 \mathrm{~A}$ load capacity and achieves a $76 \%$ efficiency. It contains a radiation tolerant ASIC with total ionizing dose up to $200 \mathrm{Mrad}$ ( $\mathrm{Si}$ ) and displacement damage up to $5 \times 10^{14} \mathrm{n} / \mathrm{cm}^{2}$. To power the L1DDC board with the two appropriate voltage levels $(2.5 \mathrm{~V}$ and $1.5 \mathrm{~V})$, two FEAST devices are used. The $1.5 \mathrm{~V}$ analog voltage for the GBTX PLLs is provided from the same FEAST device after filtering. The overall power consumption of the L1DDC is estimated to be $6 \mathrm{~W}$.

\section{Frame format}

On the fiber side the GBTX transmits frames of 120 bits in the interval of $25 \mathrm{~ns}$ (BC clock), resulting in a line rate of $4.8 \mathrm{Gbps}$. The GBTx has the ability to transmit three different types of frames, the GBT frame, the $8 \mathrm{~b} / 10 \mathrm{~b}$ frame and the wide frame.

In the GBT frame four bits are used for the frame header $(\mathrm{H})$ and 32 are used for FEC. The data transmission is thus limited to 84 bits, corresponding to a user bandwidth of $3.36 \mathrm{Gbps}$. 
From the 84 bits, the four are dedicated for Slow Control (SC) information (Internal Control (IC) and External Control (EC) fields) as outlined in Figure 7.

\begin{tabular}{|c|c|c|c|c|c|c|c|c|c|}
\hline \multicolumn{10}{|c|}{120 bits } \\
\hline r & $\begin{array}{l}\text { Internal } \\
\text { Control }\end{array}$ & $\begin{array}{l}\text { External } \\
\text { Control }\end{array}$ & & & & & & & \\
\hline $\begin{array}{c}\text { Header } \\
(3: 0)\end{array}$ & IC (1:0) & EC $(1: 0)$ & $\begin{array}{c}\text { Data } \\
(47: 64)\end{array}$ & $\begin{array}{c}\text { Data } \\
(63: 48)\end{array}$ & $\begin{array}{c}\text { Data } \\
(47: 32)\end{array}$ & $\begin{array}{c}\text { Data } \\
(31: 16)\end{array}$ & $\begin{array}{c}\text { Data } \\
(15: 0)\end{array}$ & $\begin{array}{c}\text { FEC } \\
(31: 16)\end{array}$ & $\begin{array}{c}\text { FEC } \\
(15: 0)\end{array}$ \\
\hline $\begin{array}{c}\text { Header fiel } \\
-4 \text { bits }\end{array}$ & $\begin{array}{l}\text { Slow } \\
\text { field }\end{array}$ & $\begin{array}{l}\text { ontrol } \\
\text { 4bits }\end{array}$ & & & field - 8 & bits & & $\begin{array}{l}\text { Forw } \\
\text { orrection }\end{array}$ & $\begin{array}{l}\text { Error } \\
\text { eld - 32bits }\end{array}$ \\
\hline
\end{tabular}

Fig. 7: GBTX frame format.

The FEC algorithm is built by interleaving two Reed-Solomon RS(15,11) encoded words with 4-bit symbols, each capable of correcting a double symbol error. This means that a sequence of up to 16 consecutive corrupted bits can be corrected. Finally, all configuration registers inside the GBTX ASIC are fully protected against SEUs with triple redundant registers [4].

\section{ON DETECTOR PLACEMENT}

The location of the L1DDC on the MM detectors will be in the center of both sides of the wedge (this is four planes of each detector technology) as shown in Figure 8. This provides a way of equalizing the cable length as the FE boards are radially placed on both sides of the detector.

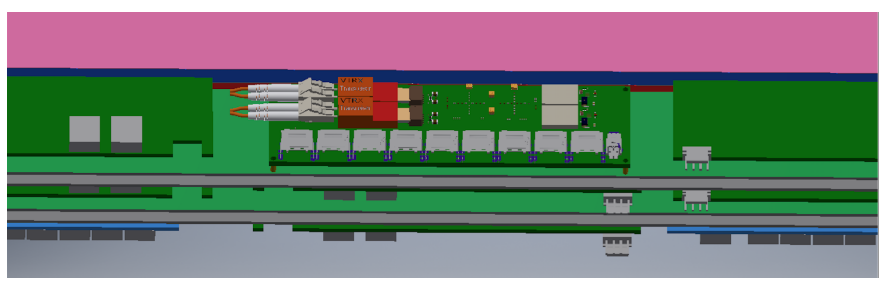

Fig. 8: FE and L1DDC placement on a MM wedge. The L1DDC boards are placed on the first and forth plane of the wedge

In this case, a single L1DDC serves the eight FE boards on one side of the plane. There are $16 \mathrm{FE}$ boards in every plane resulting in 64 per wedge. This means that eight L1DDC boards are needed for every wedge. Also, there are two wedges in every sector and there are 16 sectors in every wheel. In summary, 512 L1DDC are needed for the MM detectors and 512 for the sTGCs detectors resulting in a total 1024 of L1DDC boards [2].

All the components of the L1DDC will be placed on the top side except from the GBTX which will be placed on the bottom side of the board. An elastic thermal foam will be used to attach the bottom side of the board to the cooling channel in order to keep the heat at a low level. For the FEAST ASICs thermal pads and through vias will be used to transfer the heating to the bottom side of the board.

\section{CONCLUSION}

The L1DDC board is the intermediate board responsible to collect the Level-1 data and to distribute the TTC and
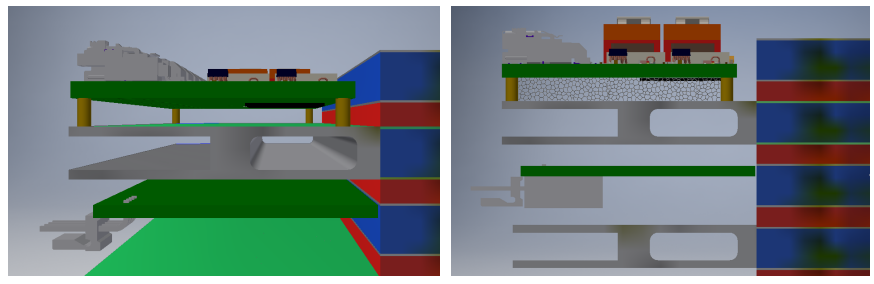

Fig. 9: A cross section of a $\mathrm{MM}$ wedge. In the upper part of the left picture, the L1DDC board placed on the detector is illustrated. All components are placed on the top side in contrast to the bottom side of the board where only the GBTx ASICs are placed. These ASICs are attached to the cooling channel with the help of an elastic thermal foam. On the left side of the cooling channel the spacer with the high and low voltage cables is illustrated. Finally, on the bottom side of the picture the FE board, placed between the two planes is also visible.

Level-1 trigger to the FE electronics. It is capable to handle a large amount of data and is fully compliant with the HLLHC rates. In addition, L1DDC uses only radiation tolerant components and is equipped with SEU mechanisms in order to ensure the signal integrity. Its dimensions are relatively small in order to fit between two readout panels of the MM and sTGC chambers of the NSW detector for the upgrade of the ATLAS experiment. Finally, the L1DDC board must have a high reliability as after the installation of the NSW it will not be accessible for replacement.

\section{REFERENCES}

[1] ATLAS Collaboration, The ATLAS Experiment at the CERN Large Hadron Collider, JINST 3 S08003, August 2008, p.4-18.

[2] ATLAS Collaboration, New Small Wheel Technical Design Report, June 2013, p.36-38, p.46-49.

[3] J Anderson, A Borga, H Boterenbrood, H Chen, K Chen, G Drake, D Francis, B Gorini, F Lanni, G Lehmann Miotto, L Levinson, J Narevicius, C Plessl, A Roich, S Ryu, F Schreuder, J Schumacher, W Vandelli, J Vermeulen, J Zhang, FELIX: a High-Throughput Network Approach for Interfacing to Front End Electronics for ATLAS Upgrades, May 2015.

[4] P. Moreira, J. Christiansen and K. Wyllie, GBTx Manual, ver 0.8, May 2015, p.9-10, p.14-21.

[5] S. Ask, D. Berge, P. Borrego-Amaral, D. Caracinha, N. Ellis, P. Farthouat, P. Glln, S. Haas, J. Haller, P. Klofver, A. Krasznahorkay, A. Messina, C. Ohm, T. Pauly, M. Perantoni, H. Pessoa Lima Junior, G. Schuler, D. Sherman, R. Spiwoks, T. Wengler, J.M. de Seixas and R. Torga Teixeiraah, The ATLAS central level-1 trigger logic and TTC system, JINST 3 P08002, August 2008, p.2-5.

[6] V. Polychronakos, L. Yao, ADDC Design Report for February 2015 NSW Electronics design reviews, ver 1.1, February 2015.

[7] G. de Geronimo, N. Ambiar, E. Vernon, N. Felt, J. Fried, G. Iakovidis, S. Li, J. Mead, J. Metcalfe, V. Polychronakos, VMM: A Front End ASIC for the Detectors of the New Small Wheels, ver 0.5, 2012.

[8] Preliminary MMFE-8 Specification, ver 0.5, February 2015.

[9] JEDEC SOLID STATE TECHNOLOGY ASSOCIATION Scalable LowVoltage Signaling for $400 \mathrm{mV}$ (SLVS-400), October 2001, p.1-6.

[10] M. Menouni, P.Gui and P. Moreira, The GBTIA, a 5 Gbit/s RadiationHard Optical Receiver for the SLHC Upgrades.

[11] GBT Project, GBTIA specifications, ver 1.7, May 2008.

[12] GBT Project, GBLD Specifications, ver 1.0, May 2015, p.3-6.

[13] Project DC-DC, FEAST Datasheet, ver 1.0, 2014, p.1-3. 